\title{
Eosinophilic Infiltrate
}

National Cancer Institute

\section{Source}

National Cancer Institute. Eosinophilic Infiltrate. NCI Thesaurus. Code C35981.

An inflammatory infiltrate composed exclusively or predominantly of eosinophils. 